\title{
ON GLOBAL SOLUTIONS FOR PARTIAL DIFFERENTIAL EQUATIONS OF FIRST ORDER ${ }^{1}$
}

\author{
BY PETER DOMBROWSKI
}

Communicated by I. M. Singer, November 28, 1960

In this note we state a theorem which guarantees the existence and uniqueness of a global solution for the Cauchy initial value problem for a complete, regular system of partial differential equations of first order for one unknown function on a manifold-all data being of class $C^{\infty}$.

This result depends on one hand on an investigation of the following problem for a $m$-dimensional $C^{\infty}$-manifold $W$, which is foliated by $r$-dimensional leaves, the leaf passing through $w \in W$ being denoted by $C_{(w)}$ : Given a $k$-dim. submanifold $A$ of $W$ with $k+r \leqq m$, find a foliated $C^{\infty}$-manifold $S$ and a $C^{\infty}$-immersion $j: S \rightarrow W$ such that (i) $j(S)=\cup_{a \in A} C_{(a)}$, (ii) each leaf of $S$ is mapped under $j$ onto a leaf of $W$. Concerning this problem we prove: If for all $a \in A$ the tangent spaces of $A$ and $C_{(a)}$ at the point $a$ have only the zero vector in common and if-in case $r>1$-for all $a \in A$ the leaf $C_{(a)}$ is simply connected, then there exist $S$ and $j$ with the desired properties. From this result and from the theory of characteristics for the above mentioned systems (essentially due to Cauchy, Lie, Goursat and E. Cartan [2] ${ }^{2}$ we obtain the announced theorem. Complete proofs and other applications will be published elsewhere.

Notations. "Manifold" will always mean a connected $C^{\infty}$-manifold which is Hausdorff and has a countable basis of open sets. If $N$ is a manifold, $N_{n}$ denotes the tangent space to $N$ at $n \in N, \mathfrak{F}(N)$ the ring of global $C^{\infty}$-functions on $N, \mathfrak{X}(N)$ the $\mathfrak{F}(N)$-module of global $C^{\infty}$-vector-fields on $N$, and a manifold $L$ is called a submanifold of $N$, if the underlying set of $L$ is a subset of the underlying set of $N$ and if the inclusion map $i: L \rightarrow N$ is a $C^{\infty}$-immersion.

DATA AND DEFINITIONS. Let $M$ be a $d$-dim. manifold and $T^{*}(M)$ its cotangent-bundle. Consider the $(2 d+1)$-dim. product-manifold $T^{*}(M) \times R$, where $R$ denotes the real line. Let $W$ be a system of $r$ partial differential equations of first order for one unknown function on $M$, i.e., $W$ is a submanifold of $T^{*}(M) \times R$ of codimension $r$. A local

1 This research was supported in part by the United States Air Force under Contract No. AF-49(638)-42, monitored by the Air Force Office of Scientific Research of the Air Research and Development Command.

${ }^{2}$ In trying to understand E. Cartan's description of the theory of characteristics and in developing a more intrinsic form of it for our special case (sketched below) a paper of M. Breuer [1] has been of help to me. 
solution for $W$ is a $C^{\infty}$-function $f$ defined on an open subset $D f$ of $M$, such that $\left.p \rightarrow d f\right|_{p} \times f(p)$ is a $C^{\infty}$-mapping of $D f$ into $W$. The sheaf of germs of local solutions for $W$ will be denoted by $s(W)$, its natural projection onto $M$ by $\sigma .^{3}$ If $s \in \mathcal{S}(W)$ we define the integral element of $s$ to be $\iota(s):=\left.d f\right|_{p} \times f(p)$ with $p=\sigma(s)$ and $f$ being a local solution representing $s$; thereby we obtain a unique map $\iota: \delta(W) \rightarrow T^{*}(M) \times R$. A consideration of well-known examples shows (compare, e.g., the imaginary part of the complex logarithm) that it is desirable to extend the concept of a solution in such a way that the range of definition of a solving function is not necessarily an open subset of $M$ but a "domain over $M$ ": A triple $(D, j, f)$ consisting of a $d$-dim. manifold $D$, an immersion $j$ of $D$ into $M$ and $f \in \mathcal{F}(D)$ is called a solution for $W$ if for each open subset $G$ of $D$ for which $j \mid G$ is diffeomorphic, the function $f \circ(j \mid G)^{-1}$ is a local solution for $W$. If $(D, j, f)$ is a solution for $W$, then each point $p \in D$ has a neighborhood $U_{p}$ such that $j \mid U_{p}$ is diffeomorphic. Henceforth there exists a unique mapping $f_{j}: D$ $\rightarrow S(W)$ which assigns to each $p \in D$ the germ of the local solution $f \circ\left(j \mid U_{p}\right)^{-1}$ at $j(p)$.

Consider now the following Cauchy initial value problem.

(P): Given $a(d-r)$-dim. submanifold $A$ of $M$ and $g \in \mathcal{F}(A)$, find $a$ solution $(D, j, f)$ for $W$ and a 1-1 immersion $k$ of $A$ into $D$ such that $f \circ k=g$.

We call a solution $(D, j, f), k: A \rightarrow D$ of the problem (P) unique for every $\tilde{f} \in \mathcal{F}(D)$, for which $(D, j, \tilde{f}), k: A \rightarrow D$ is a solution of problem (P) too, it follows $\tilde{f}=f$.

In order to introduce the concept of a "global" solution" of $(\mathrm{P})$ we have to review the theory of characteristics for $W$ :

Let $\pi: T^{*}(M) \rightarrow M, \psi: T^{*}(M) \times R \rightarrow T^{*}(M), z: T^{*}(M) \times R \rightarrow R$ denote the natural projections and define $\phi:=\pi \circ \psi$. On the manifold $T^{*}(M) \times R$ we have the following natural Pfaffian form $\nu$ : If $a:=(\omega \times \alpha) \in T^{*}(M) \times R$ and $X \in\left(T^{*}(M) \times R\right)_{a}$ then

$$
\nu(X):=d z(X)-\omega\left(\phi_{*}(X)\right) .
$$

${ }^{3}$ Since $\sigma(\mathcal{S}(W))$ is open in $M$, one can assume without loss of generality that $\sigma$ is surjective.

4 The most immediate idea, to call a solution of $(\mathrm{P}):(D, j, f), k: A \rightarrow D$ a "global" one, would be to ask that $f_{j}(D)$ is a connected component of $\boldsymbol{S}(W)$. But under the natural hypothesis that $W$ is complete and regular (see below) one finds that every connected component of $\mathcal{S}(W)$ is a (non-Hausdorff) topological space containing a noncountable family of pairwise disjoint open sets. The latter fact excludes that $f_{i}(D)$ equals any connected component of $S(W)$. Our proposal to define "globality" is inspired by the concept of the "characteristic field" for one linear partial differential equation of first order, introduced by E. Kamke [3]. 
For $f \in \mathcal{F}\left(T^{*}(M) \times R\right)$ we define gradient $(f)$ to be the unique vectorfield $\nabla f$ on $T^{*}(M) \times R$ such that

$$
\begin{aligned}
& \nu(\nabla f)=0 \text { and } d \nu(X, \nabla f)=d f(X) \\
& \quad \text { for all } X \in \mathfrak{X}\left(T^{*}(M) \times R\right) \text { with } \nu(X)=0 .
\end{aligned}
$$

If $f, g \in \mathcal{F}\left(T^{*}(M) \times R\right)$, the function $[f, g]:=d \nu(\nabla f, \nabla g)$ is called the Jacobi-bracket of $f$ and $g$. (One proves for $f, g, h \in \mathcal{F}\left(T^{*}(M) \times R\right.$ ):

$$
\begin{aligned}
(\nabla[f, g]-[\nabla f, \nabla g])(h) & =[[f, g], h]+[[g, h], f]+[[h, f], g] \\
& =[f, g] \frac{\partial h}{\partial z}+[g, h] \frac{\partial f}{\partial z}+[h, f] \frac{\partial g}{\partial z},^{\mathbf{5}}
\end{aligned}
$$

where $[\nabla f, \nabla g]$ means the Lie-bracket of $\nabla f, \nabla g$.

$W$ is called complete if for all $f, g \in \mathfrak{F}\left(T^{*}(M) \times R\right)$ and for each open subset $G$ of $W$ the following holds: If $f|G=g| G=0$, then $[f, g] \mid G=0$. $W$ is called regular if the map $(\phi \times z) \mid W: w \rightarrow \phi(w) \times z(w)$ of $W$ into $M \times R$ is everywhere on $W$ of rank $d+1$. For $w \in W$ we define

$$
C_{w}:=\left\{\left.\nabla f\right|_{w} \mid f \in \mathfrak{F}\left(T^{*}(M) \times R\right) \text { and }\left.d(f \mid W)\right|_{w}=0\right\} .
$$

One checks immediately that $C_{w}$ is a subspace of $W_{w}$-after identifying $W_{w}$ in the canonical way with the subspace $i_{*}\left(W_{w}\right)$ of $\left(T^{*}(M) \times R\right)_{w}$, where $i: W \rightarrow T^{*}(M) \times R$ is the inclusion map. For the integration theory the following description of $C_{w}$ (essentially due to E. Cartan) is useful:

$$
\begin{aligned}
C_{w}=\left\{X \mid X \in W_{w} \text { and } \nu(X)=d \nu(X, Y)=0\right. \\
\text { for all } \left.Y \in W_{w} \text { with } \nu(Y)=0\right\} .
\end{aligned}
$$

If $W$ is complete and regular, one proves that $\left\{C_{w}\right\}_{w \in W}$ forms an $r$-dim. involutive distribution on $W$, the maximal (connected) integral manifolds of which are called the characteristic submanifolds of $W$. The characteristic submanifold passing through $w \in W$ will be denoted by $C_{(w)}$. Its tangent space at $w$ will be identified with $C_{w}$.

Assume now that $W$ is complete and regular: If $S$ is an arbitrary subset of $s(W)$ we call the characteristic hull of $S$ the set of all $s \in \mathcal{S}(W)$ with the following property: There exists a continuous map $c:[0,1] \rightarrow S(W)$ with $c(0) \in S$ and $c(1)=s$ and such that $\iota \circ c:[0,1]$ $\rightarrow W$ is a map into one of the characteristic submanifolds of $W$.

\footnotetext{
5 The second of these two equations is due to A. Mayer [4] and proves that $[\cdots, \cdots]$ turns not $\mathcal{F}\left(T^{*}(M) \times R\right)$ but the subring of $\mathcal{F}\left(T^{*}(M) \times R\right)$ consisting of the functions $f$ with $\partial f / \partial z=0$ into a Lie-algebra over $R$. (Observe: $(\partial / \partial z)[f, g]$ $=[\partial f / \partial z, g]+[f, \partial g / \partial z]$.)
} 
A solution $(D, j, f)$ for $W$ is called global, if $f_{j}(D)$ coincides with its characteristic hull. ${ }^{6}$

We state now the main result:

Theorem. Let $M$ be $a d$-dim. manifold, $W$ a submanifold of $T^{*}(M)$ $\times R$ of codim. $r, r \in\{1, \cdots, d\}$. Let $A$ be $a(d-r)$-dim. submanifold of $M$ and $g \in \mathcal{F}(A)$.

Hypothesis: (i) $W$ is complete and regular.

(ii) There exists a $(d-r)$-dim. submanifold $B$ of $W$ such that

(a) $B$ is an integral manifold of $\nu$,

(b) $\phi \mid B$ is a diffeomorphism of $B$ onto $A$ and $z \circ(\phi \mid B)^{-1}=g$,

(c) for all $b \in B$ the map $\phi \mid C_{(b)}: C_{(b)} \rightarrow M$ is transversal to $A$ in $b$, i.e., $\phi_{*}\left(C_{b}\right)$ and $A_{\phi(b)}$ have no nonzero tangent vector in common.

(iii) If $r>1$, then for all $b \in B$ the manifold $C_{(b)}$ is simply connected.

Claim: There exists a global solution $(D, j, f)$ for $W$ and a 1-1 immersion $k$ of $A$ into $D$ such that $f \circ k=g$. If furthermore $\tilde{f} \in \mathfrak{F}(D)$ such that $(D, j, \tilde{f})$ is a solution for $W$ and $\tilde{f} \circ k=g$, then $\tilde{f}=f$.

\section{BIBLIOGRAPHY}

1. M. Breuer, Jacobische Differentialgeometrie und Systeme partieller Differentialgleichungen erster Ordnung, Bonn. Math. Schr. no. 7 (1958).

2. E. Cartan, Les systèmes différentielles extérieurs et leurs applications géométriques, Paris, Hermann, 1945.

3. E. Kamke, Differentialgleichungen reeller Funktionen, Leipzig, 1930, p. 317.

4. A. Mayer, Math. Ann. vol. 9 (1876) p. 370.

\section{Massachusetts Institute of Technology}

- This concept of "globality" becomes intuitively more clear by pointing out its striking analogy to the construction of Riemann surfaces by means of the process of analytic continuation of germs of holomorphic functions along paths. In general there is, depending on the fact that we are in the nonanalytic case, no unique procedure to "continue" a germ of local solutions for $W$ along an arbitrary path in $M$, thereby causing the "misbehavior" of the sheaf $S(W)$ described in footnote 4 . But if we take a path in $M$, which is "characteristic," i.e., the path is the projection under $\phi$ of a path in a characteristic submanifold of $W$, then there exists (even in the $C^{\infty}$ case), if $W$ is complete and regular, at most one continuation of a germ of local solutions for $W$ along such a characteristic path. Then a solution $(D, j, f)$ is global iff for every germ $s$ of local solutions for $W$ which is "realized" by $(D, j, f)$, (i.e., $s \in f_{j}(D)$ ), all germs of local solutions for $W$ are realized by $(D, j, f)$ too, which can be obtained by the continuation of $s$ along characteristic paths in $M$.

${ }^{7}$ From (i) follows that $\phi \mid C_{(b)}$ is an immersion. 